\title{
Combating Biofilm by a Self-Adapting Drug Loading Hydrogel
}

Xia Li, ${ }^{a}$ Ya-nan Fu, ${ }^{a, c}$ Lifei Huang, ${ }^{a}$ Fang Liu, ${ }^{b}$ Thomas Fintan Moriarty, ${ }^{c}$ Lei Tao, ${ }^{d}$ Yen Wei, ${ }^{d}$ and Xing Wang*,a

${ }^{a}$ Beijing Laboratory of Biomedical Materials, Beijing University of Chemical Technology, Beijing 100029, P. R. China

${ }^{b}$ Department of Gastroenterology, China-Japan Friendship Hospital, Beijing 100029, P. R. China

${ }^{c}$ AO Research Institute Davos, Clavadelerstrasse 8, 7270 Davos Platz, Switzerland ${ }^{d}$ The Key Laboratory of Bioorganic Phosphorus Chemistry \& Chemical Biology (Ministry of Education), Department of Chemistry, Tsinghua University, Beijing 100084, China

*E-mail: wangxing@mail.buct.edu.cn 


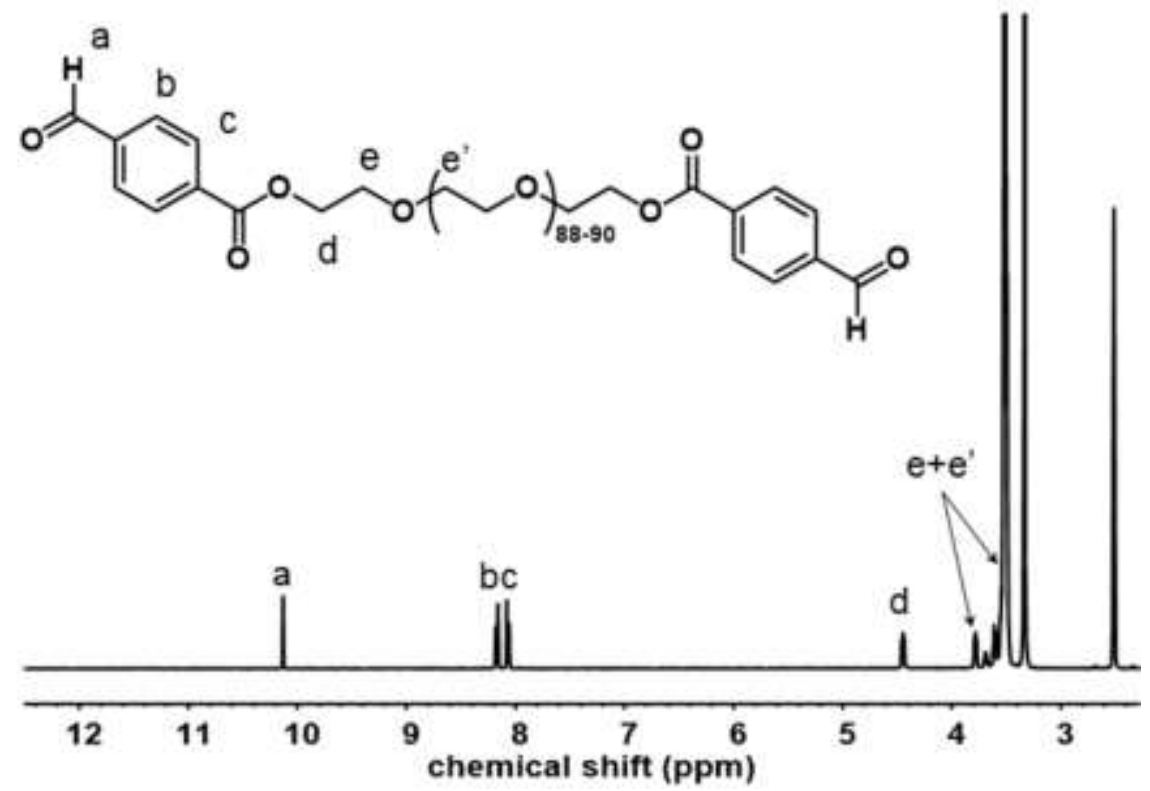

Figure S1. The ${ }^{1} \mathrm{H}$ NMR spectrum of DF-PEG 4000 .

a

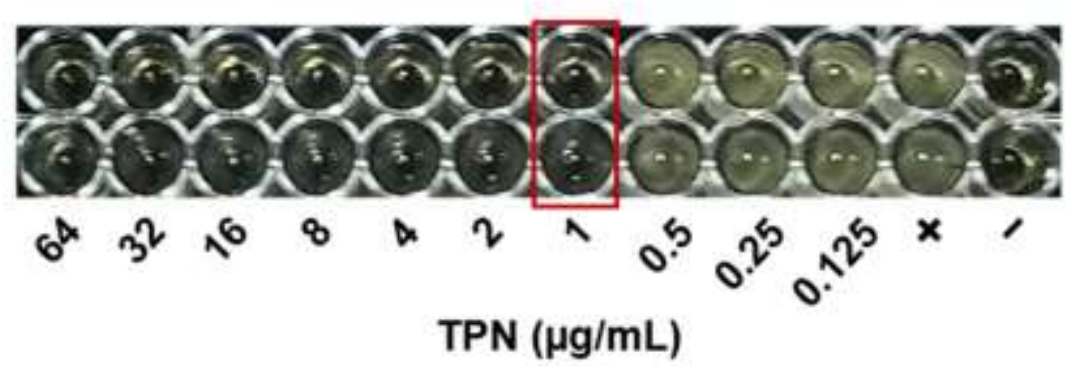

b

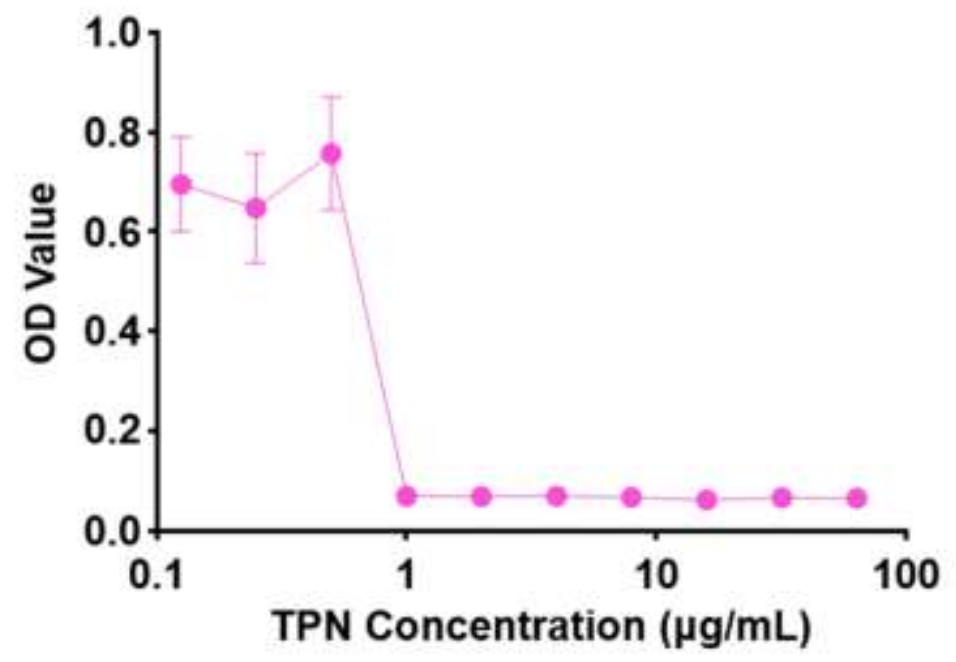

Figure S2. a. Minimum inhibitory concentration (MIC) test of TPN. b. Quantitative results of MIC test of TPN ( $\mathrm{n}=3$ for each group). 

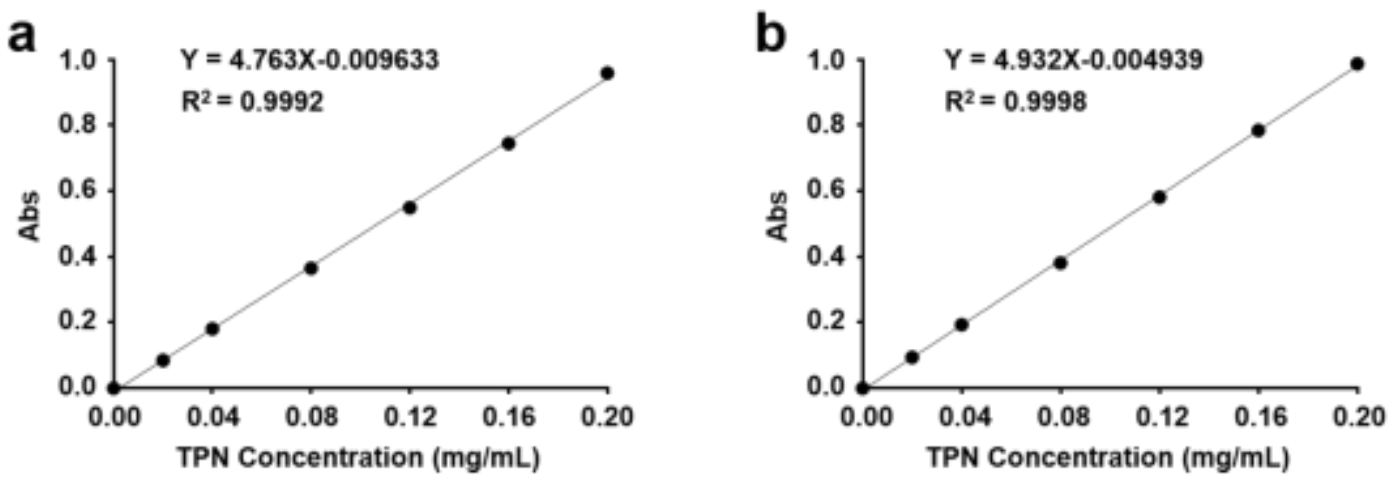

Figure S3. The standard curves of TPN in PBS with pH 5.4 (a) and PBS with $\mathrm{pH} 7.5(\mathbf{b})$, respectively.

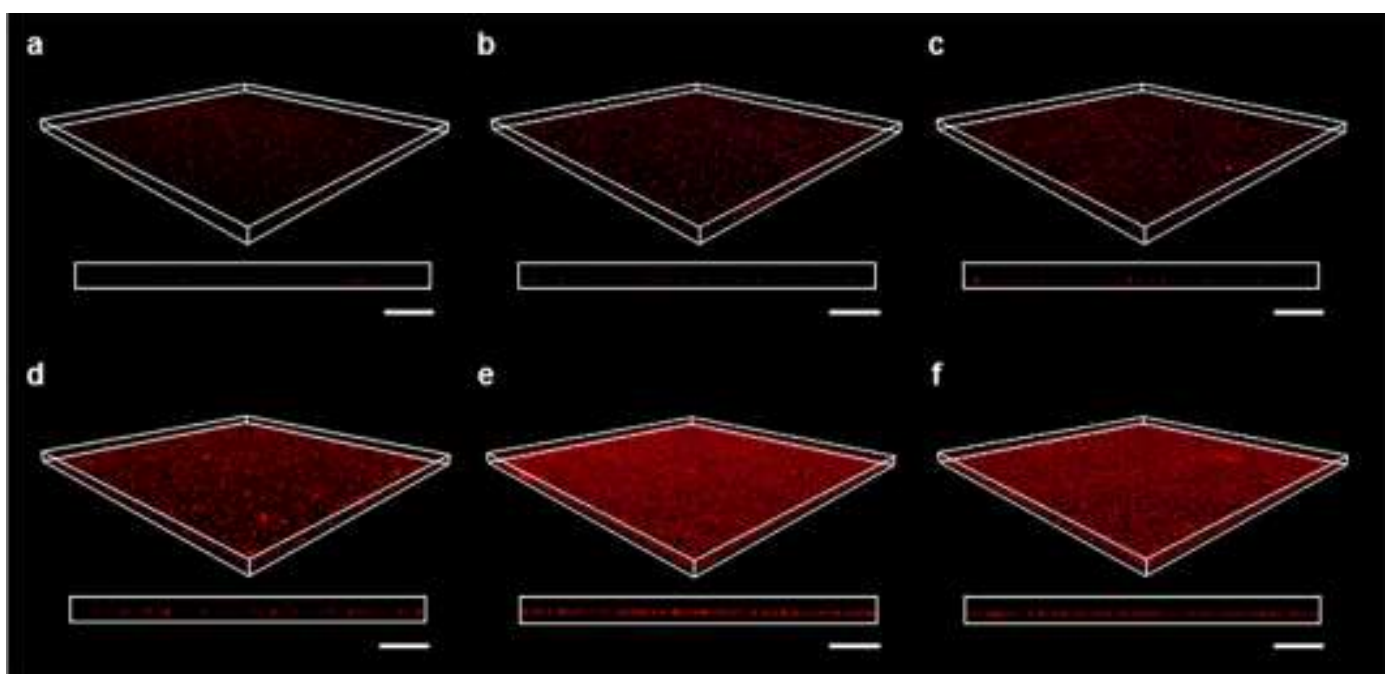

Figure S4. Combating effect of PBS (a), CPH (b), CPH1 (c), TPN (d), TPN-CPH (e) and TPN-CPH1 (f) on $S$. aureus biofilm in vitro. The bar is $50 \mu \mathrm{m}$. CPH contains $0.25 \mathrm{wt} \%$ DF-PEG, and CPH1 contains 0.5wt\% DF-PEG.

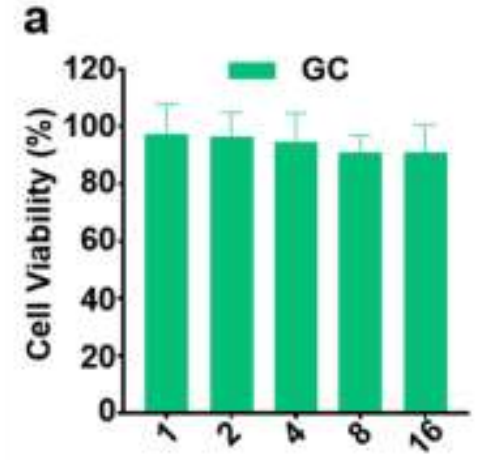

Concentration $(\mathrm{mg} / \mathrm{mL})$
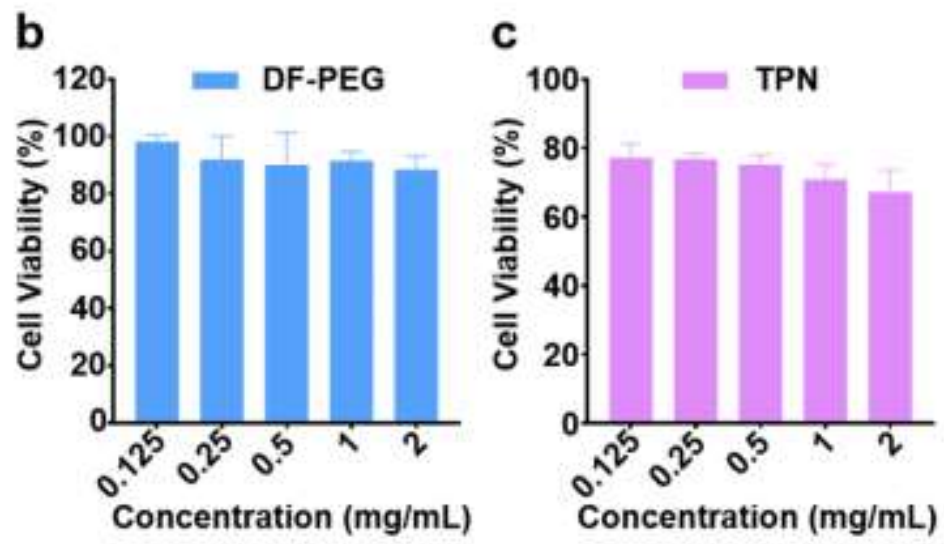

Figure S5. Cytotoxicity evaluation of GC (a), DF-PEG (b), TPN (c) to L929 cells ( $\mathrm{n}=$ 4 for each group). 


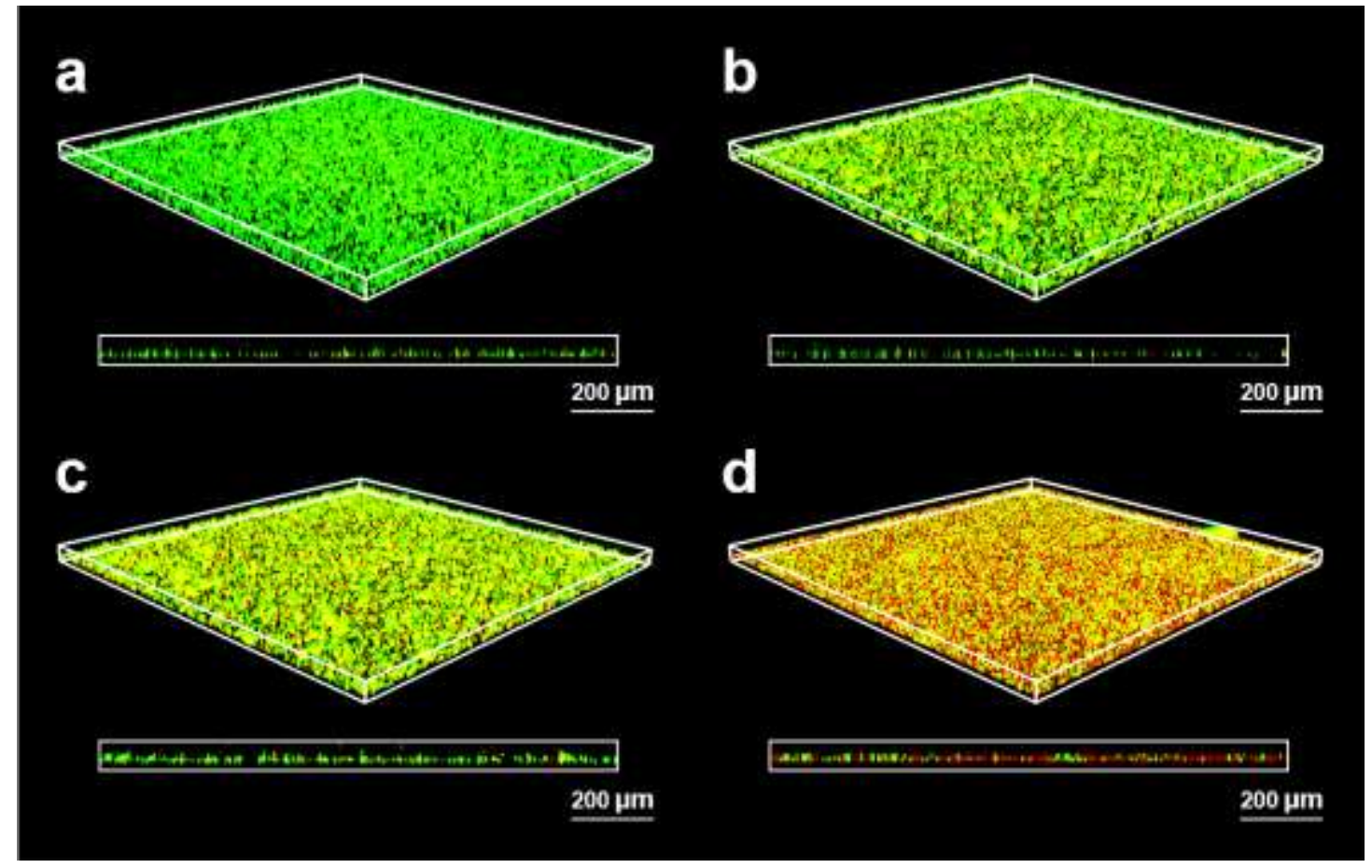

Figure S6. Living and dead bacteria distribution in biofilms treated with (a) PBS, (b) CPH, (c) TPN and (d) TPN-CPH. Green: SYTO 9; Red: PI. 